\title{
Copper-Catalyzed Asymmetric Synthesis of Piperidines by a [6+3] Cycloaddition
}

\section{Category}

Metal-Catalyzed

Asymmetric

Synthesis and

Stereoselective

Reactions

\section{Key words}

azomethine ylides

copper

cycloaddition

heterocycles<smiles></smiles>

$\mathrm{Cu}(\mathrm{MeCN})_{4} \mathrm{BF}_{4}(5 \mathrm{~mol} \%)$ (S)-TF-BiphamPhos (6 mol\%)

$\mathrm{Cs}_{2} \mathrm{CO}_{3}, \mathrm{CH}_{2} \mathrm{Cl}_{2},-40{ }^{\circ} \mathrm{C}$

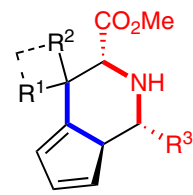

25 examples up to $92 \%$ yield up to $94 \%$ ee dr up to $>20: 1$

$R^{1}=R^{2}=$ cyclobutyl, cyclopentyl, Cy,

cycloheptenyl, tetrahydropyranyl, Et

dr up to $>20: 1$
$(0.25 \mathrm{mmol}$ scale $)$

$\mathrm{R}^{1}=\mathrm{H}$

$\mathrm{R}^{2}=\mathrm{Ph}, 4-\mathrm{ClC}_{6} \mathrm{H}_{4}, \mathrm{PMP}, \mathrm{Et}$

$\mathrm{R}^{3}=\mathrm{Ph}, 4-\mathrm{ClC}_{6} \mathrm{H}_{4}, \mathrm{PMP}, 4-\mathrm{BrC}_{6} \mathrm{H}_{4}$

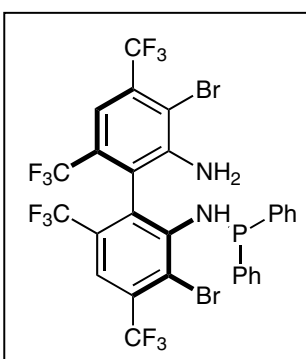

(S)-TF-BiphamPhos

Selected examples:<smiles>CC(=O)[C@H]1N[C@@H](c2ccc(Cl)cc2)[C@@H]2C=CC=C2C12CCC2</smiles>

Selected further transformations:<smiles>COc1ccc(C2C3=CC=CC3[C@@H](c3ccc(Cl)cc3)N[C@H]2C(C)=O)cc1</smiles><smiles>CCC1C2=CC=CC2[C@@H](c2ccc(Cl)cc2)N[C@@H]1C(C)=O</smiles>

$55 \%$ yield $91 \%$ ee $\mathrm{dr}=4: 1$<smiles>CC(=O)[C@H]1N[C@@H](c2ccc(Cl)cc2)C2CCCC2C1(C)C</smiles><smiles>C[Te]C[Te]</smiles>
$\mathrm{CO}_{2} \mathrm{Me}$<smiles>C[C@H]1NCC2C=CC=C2C1(C)C</smiles><smiles>Cc1ccc(Cl)cc1</smiles><smiles>CN1C(=O)C=CC1=O</smiles><smiles>CC(=O)[C@H]1N[C@@H](c2ccc(C)cc2)C2C=CC=C2C1(C)C</smiles>

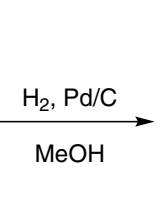<smiles>CC(=O)[C@H]1N[C@@H](c2ccc(Cl)cc2)C2CCC=C2C1(C)C</smiles>

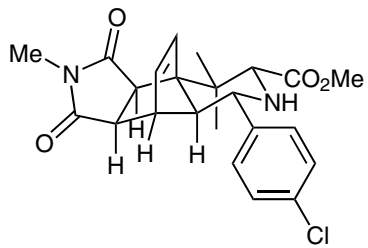

Significance: Hong et al. (Org. Lett. 2003, 5, 1689) reported a [6+3] cycloaddition of azomethine ylides with fulvenes, thus leading to the synthesis of racemic six-membered piperidine derivatives. However, there is a lack of catalytic asymmetric variants with high functional group tolerability.
Comment: A highly efficient asymmetric copper(I)-TF-BiphamPhos-catalyzed [6+3] cycloaddition was developed, which shows very good yields, high regioselectivity and excellent enantioselectivity. Due to its high functional group tolerance, the products can be easily modified.

SYNFACTS Contributors: Mark Lautens, Harald Weinstabl 